\title{
Membranes stay afloat
}

\section{The difficulty in working with lipids and other membrane components has left many fundamental questions about the biochemistry of membranes unanswered. New techniques are required to determine how cell membranes are organized structurally and functionally.}

$T^{\mathrm{T}}$ he bad reputation of lipids goes beyond the dangers of the lowdensity lipoprotein complex LDL and its associated artery-clogging cholesterol. It extends to the research laboratories and to anyone who has ever tried to work with membrane proteins. Indeed, the purification and subsequent crystallization of the protein-conducting channel SecY was not achieved until two decades after its discovery (Nature 427, 36-44, 2004). If you aren't working on membrane proteins, you might be able to avoid thinking about lipids (particularly if you work with prokaryotes), but studying eukaryotic cells requires that you pay attention to their organization and compartmentalization in lipid-bound organelles. In this issue, we spotlight a range of articles that speak to the problem of studying membranes as well as the progress that has been made in understanding the biochemistry, biophysics and cell biology of these ubiquitous structures.

The plethora of membrane events that happen within eukaryotic cells extends beyond the cell biology and membrane trafficking events that bring proteins from where they are formed to where they ultimately function. It reaches to fission and fusion events in which chemical and biophysical interactions of individual lipids and proteins can dictate the organization of signaling events from membrane platforms. In a historical look at the field of membrane traffic, an interview with Randy Schekman highlights the importance that traditional approaches have had in developing our current understanding of protein transport via tiny membrane vesicles (p. 568).

Commentaries by both Gerald Feigenson (p. 560) and Joshua Zimmerberg (p. 564) point out how far away we are from modeling a real, intact membrane with its full complement of lipids and proteins. Feigenson calls for modeling these membranes based on simple systems with just one representative lipid from each of the three main lipid groups. Given the hundreds of different lipid types found in a cell membrane, Zimmerberg argues that an understanding of the chemical and physical nature of individual lipids should be integrated with approaches that treat a membrane as a continuum. From a chemical perspective, both Commentaries argue for decoding the information from the structure of lipids (for example, their chain length and double-bond patterns) to build a model for how intact membranes are organized and function.

The organizational complexity of membranes becomes evident when one considers that there is dedicated machinery for moving membrane lipids between the outer leaflets of different membrane compartments. A Perspective by Vytas Bankaitis defines a place for phosphatidylinositol transfer proteins in lipid metabolism and signaling as part of a "nanoreactor" that integrates lipid metabolism and signaling with membrane traffic (p. 576). Another level of membrane complexity is demonstrated by the lipidation of proteins, which allows otherwise soluble proteins to associate with membranes. As reviewed by Marilyn Resh, this type of modification is particularly important for many signaling proteins that do not need to remain in the membrane, or that may actually need to shuttle between compartments to function properly (p. 584).

Two primary research articles in this issue interrogate membrane proteins to demonstrate the relationship between their structure and function. Yoshida et al. show that nitric oxide regulates cellular $\mathrm{Ca}^{2+}$ flux through S-nitrosylation of specific cysteine residues on transient receptor potential cation channels (p. 596), and Hong et al. show that the alternate pairing of a glutamate with either a neighboring arginine or lysine dictates whether OmpA is open or closed (p. 627). In addition, we report on a recent article that identifies new machinery for integrating membrane proteins of Escherichia coli into membranes (p. 575).

Clearly, the fields of lipid and membrane research have benefited from diverse techniques and disciplines. For instance, from work on model membranes within the past decade, we have come to appreciate that even within a single membrane, there are "raftophilic" lipids that associate into distinct membrane domains. The fact that there is a long-standing debate about the existence of such raft domains points at the difficulty of working with lipids and membranes and warrants a call to action for new techniques to be developed. These techniques should include those that help us to get a better idea of what constitutes a bilayer membrane at its most basic chemical and biophysical level and those that help us to visualize these nebulous membrane domains in intact cells. Tools of the biochemist, the biophysicist and the cell biologist will be required to achieve a mechanistic look at any membrane event. For instance, on p. 575 we direct you to a new secondary-ion mass spectrometry method that shows promise in advancing our understanding of membrane composition and domain organization. Also, in a primary research article in this issue, Schlee et al. demonstrate that new combinations of experimental and mathematical-modeling approaches can be used to understand the assembly of complex multicomponent cell membrane signaling systems (p. 636). Certainly, these fields will continue to benefit greatly from thinking beyond classical strategies.

This month, Nature Chemical Biology will bring together chemists and biologists who study membrane composition and membrane events to speak in a dedicated session of our inaugural annual symposium. This conference, entitled "The Chemical Biology of the Cell," will take place in Boston and will also include sessions on the nucleus and cell division, metal ions and metabolites, and cytoplasmic processes (http:// www.nature.com/nchembio/meetings/2006symposium). Keynote talks delivered by a chemist and a biologist will provide perspectives on the future of cell and chemical biology. We hope that by bringing diverse scientists together, we will advance our thinking about membranes and show how chemical biologists may offer new insights into questions throughout cell biology. 